ARTICLE OPEN

\title{
In-situ monitoring of seeded and unseeded stage III corrosion using Raman spectroscopy
}

\author{
Joseph V. Ryan ${ }^{1}$, Benjamin Parruzot $\mathbb{D}^{1}$, Amanda M. Lines ${ }^{1}$, Samuel A. Bryan ${ }^{1}$, Lorraine M. Seymour ${ }^{1}$, Jeffrey F. Bonnett ${ }^{1}$ and \\ Radha K. Motkuri iD ${ }^{1}$
}

Stage III glass dissolution, the occasionally observed tendency for accelerated aqueous corrosion after extensive time spent at low reaction rates, is a major area of technical uncertainty in long-term glass performance modeling for vitreous nuclear waste forms. Stage III dissolution behavior is thought to be caused by the precipitation of zeolite phases that lower the activity of the ratecontrolling orthosilicic acid ion and cause the acceleration of glass dissolution. The uncertainty lies mainly in a poor understanding of the growth kinetics of these key crystalline phases. It is particularly important to understand the impact of temperature and $\mathrm{pH}$ conditions on Stage III behavior. Accurate analysis of this phenomenon is complicated by the typically very long (e.g., years), and variable length of the induction period preceding the onset of Stage III behavior. In this study of a high-sodium aluminoborosilicate glass, we demonstrate the ability to initiate the onset of Stage III behavior in a controllable fashion so as to both reduce the time delay of the incubation period, and to also be able to initiate Stage III dissolution in controlled conditions. In this case, we demonstrate that Stage III glass corrosion behavior is possible at $70^{\circ} \mathrm{C}$, a lower temperature than previously observed $\left(90^{\circ} \mathrm{C}\right)$, even in an unseeded experiment. We confirm, through a comparison study, that seeded and unseeded Stage III corrosion rates are equivalent. This enables wide-scale testing of Stage III corrosion rates for many glass compositions in relatively short times through this technique. We also report further development of the use of in situ Raman spectroscopy monitoring of boron concentration and $\mathrm{pH}$ through simple and chemometric analysis methods.

npj Materials Degradation (2019)3:34; https://doi.org/10.1038/s41529-019-0095-0

\section{INTRODUCTION}

The greatest potential uncertainty for long-term glass dissolution remains based in so-called Stage III dissolution behavior. ${ }^{1}$ Stage III dissolution behavior (Fig. 1) refers to a delayed acceleration of glass corrosion rates. This behavior is observed for most sodium aluminoborosilicate glasses that are exposed to long-term static dissolution tests run in aggressive conditions [e.g., high $\left(\geq 90^{\circ} \mathrm{C}\right)$ temperature, high ratio of surface area to volume, high $(>10.5)$ $\mathrm{pH}$, and long durations (times vary from weeks to decades). ${ }^{2}$ Some of the secondary phases that commonly form on corroding glasses consist of semi-crystalline phyllosilicate minerals ${ }^{3}$ or amorphous polymorphs with similar compositions. These mostly form at the interface between the gel layer and solution and appear to have a relatively small impact on the corrosion rate. With the precipitation of certain phases, however, the marked acceleration in the reaction rate that defines Stage III dissolution behavior can occur. Aside from static tests, certain other laboratory conditions such as those in the Pressurized Unsaturated Flow (PUF) test have also been shown to lead to Stage III behavior. ${ }^{4}$ While it appears that certain glass compositions do not intrinsically drive Stage III behavior, ${ }^{5}$ some can be forced into it when the solution is artificially altered. ${ }^{6}$

In general, Stage III behavior coincides with the formation of key secondary phases on the outer surface of the growing alteration layers. It is believed that these alteration phases have relatively fast formation kinetics and serve as a sink for orthosilicic acid, accelerating glass dissolution. ${ }^{7,8}$ Although not all of the following need to be understood to produce an acceptable calculation of performance, the uncertainty in occurrence and kinetics of Stage III behavior is multifaceted, including:

- whether or not the acceleration will occur,

- whether or not there is a correlation between glass composition and susceptibility to Stage III behavior,

- which solution and environmental conditions (temperature, $\mathrm{pH}$, etc.) will make Stage III more or less likely to occur,

- the precise identity of the critical phase(s) whose formation results in Stage III dissolution behavior,

- the duration at Stage II before Stage III dissolution behavior occurs, and

- the rate of alteration after the resumption.

Currently, data on Stage III dissolution behavior are quite limited. This is mostly because of the long and difficult to predict incubation time necessary to form secondary phases that cause Stage III behavior. The ALTGLASS ${ }^{\text {TM }}$ database (v2.1) ${ }^{9}$ is the most extensive collection of static (or near-static) dissolution test data for sodium aluminoborosilicate glasses to date. A study of this database revealed that, out of 127 static alteration tests with sufficient duration ( $>1$ year unless Stage III observed earlier), more than $70 \%$ exhibit a delayed rate acceleration (Stage III behavior), including $10 \%$ where solution data showed that the glass was entirely altered. All of the studies identified were performed at $90^{\circ} \mathrm{C}$ (or higher) and surface-area-to-volume ratios (SA/V) of $2000 \mathrm{~m}^{-1}$ (or higher) over durations from 0.5 to 9 years (generally

${ }^{1}$ Pacific Northwest National Laboratory, Energy and Environment Directorate, Richland, WA 99352, USA

Correspondence: Joseph V. Ryan (joe.ryan@pnnl.gov)

Received: 6 October 2017 Accepted: 6 August 2019

Published online: 16 October 2019 


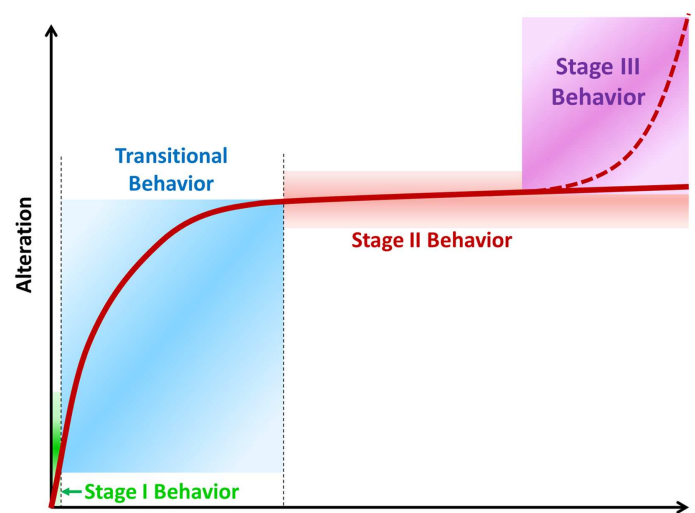

Time

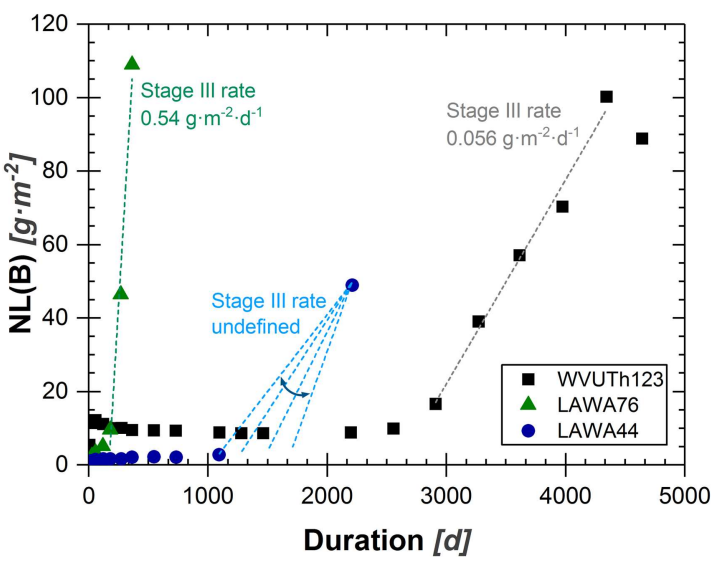

Fig. 1 Schematic (top) and experimental (bottom) depiction of glass corrosion behavior. Stage I and the transition to Stage II occur too rapidly to observe in the experimental datasets. Data are from the ALTGLASS database. ${ }^{9}$

1-5 years). Although it is very valuable to show the extent of Stage III occurrence, data from these tests have various degrees of usability in terms of examining Stage III behavior. Examples of these data are shown in Fig. 1b, where normalized mass loss calculated from boron solution data is reported as a function of time. Because the time periods are so long, it is impractical to sample the experiments frequently. Thus, when Stage III does eventually occur, the sample coverage of the event and the subsequent dissolution rate is poor at best, often including only two data points, e.g., LAWA44 data in Fig. 1b. When more data points are available during the accelerated rate period, it is possible to estimate an alteration rate, e.g., LAWA76 and WVUTh123 data in Fig. 1b.

The purpose of the experiments presented in this paper was to intentionally induce Stage III behavior by seeding tests that are in Stage II (residual rate conditions) with zeolites and measuring Stage III release rates. This allows the interrogation of environmental impacts such as temperature without the long and indeterminate incubation time that frustrates most studies of Stage III. Zeolite seeding has been attempted several times, ${ }^{10,11}$ but was not successful until recent work by Fournier. ${ }^{12}$ The key difference was in the type of zeolite used for the experiment. Fournier made a very careful characterization of a glass immediately after Stage III initiation and noted the presence of a metastable zeolite known as Na-P2 with the composition $\mathrm{Na}_{4} \mathrm{Al}_{4} \mathrm{Si}_{12} \mathrm{O}_{32} \cdot 14 \mathrm{H}_{2} \mathrm{O}$.

To help with the experimental coverage, in situ monitoring with Raman spectroscopy was used. This technique has enabled the tracking of corrosion experiments daily or even more frequently.
The Raman probe was enclosed within a stainless steel sleeve with a quartz optical window allowing direct solution measurement of the Raman scattering spectrum in a non-invasive manner. A database was constructed from the spectra of solutions systematically varied in $\mathrm{pH}$ and boric acid/borate concentrations. These spectra were used to create a chemometric model based on a multivariate analysis of the spectra. This model was then used to determine the $\mathrm{pH}$ and borate concentrations of solutions during glass dissolution experiments.

\section{RESULTS}

Unseeded dissolution tests

Prior static tests at $90^{\circ} \mathrm{C}$ show that LAWA76 glass exhibited Stage III behavior within months of the start of the test. ${ }^{9}$ Here, the solution analysis appeared to exhibit a linear or second-order increase in dissolution rate with time that started immediately upon the experiment start (Fig. 2a). The tracking of this experiment with on-line Raman monitoring (Fig. 3), however, showed that this interpretation is incorrect. While the simplified tracking algorithm was not sufficient to relate Raman intensity to the solution concentrations quantitatively, it was able to qualitatively show the evolution of the solution concentration over time. With the added temporal resolution of the Raman system, it can be seen that a rapid short-term dissolution rate transitioned into a typical Stage II residual rate that then persisted from roughly days 35-70. Thereafter, the dissolution abruptly accelerated to what appeared to be a relatively constant dissolution rate. This difference clearly illustrates the added benefits that more frequent monitoring can provide. With the understanding of the time period where Stage III was occurring, the solution analysis data points in that region were used to calculate a range of possible Stage III dissolution rates for this system between $0.40 \mathrm{~g} \mathrm{~m}^{-2} \mathrm{~d}^{-1}$ and $0.56 \mathrm{~g} \mathrm{~m}^{-2} \mathrm{~d}^{-1}$, depending on which range of data points is selected. The rate observed was a little less than one order of magnitude slower than the limiting intrinsic rate for these temperature and $\mathrm{pH}$ conditions.

The monitoring in Fig. 3 also showed one other interesting effect. The Raman signal abruptly decreased at day 155 . Upon investigation, it was found that the probe window, as well as every other submerged surface, had been coated in zeolite. Once the window was wiped clear of the material, the signal returned to expected values. This process was performed several times, leading to the jagged appearance of the dataset. Following test termination, the zeolite was collected and determined to be analcime $\left[\mathrm{NaAlSi}_{2} \mathrm{O}_{6} \cdot \mathrm{H}_{2} \mathrm{O}\right]$ following examination by X-ray diffraction. This was consistent with results from Fournier et al. that observed the transformation of zeolite Na-P2 to analcime ${ }^{13}$ in concert with seeded glass dissolution studies. ${ }^{8}$ The fact that the zeolites precipitated throughout the system is strong evidence to support the idea that they impact glass dissolution through a solution-affinity feedback mechanism.

While Stage III has been observed in most aggressive multi-year static dissolution tests of sodium aluminoborosilicate glasses, ${ }^{9}$ there is no available data for the occurrence of Stage III at temperatures below $90^{\circ} \mathrm{C}$. We initiated and monitored a test at $70{ }^{\circ} \mathrm{C}$ to see if the acceleration would happen at that temperature as well. Given the rapid transition to Stage III exhibited by A76 at $90^{\circ} \mathrm{C}$, it was thought that this system may be susceptible to the same processes at lower temperatures. After $\sim 75$ days, Stage III behavior was observed and continued in a constant fashion for over 4 months (Fig. 2b). This long period of record resulted in a measured Stage III rate of $0.13 \mathrm{~g} \mathrm{~m}^{-2} \mathrm{~d}^{-1}$ using solution analyses.

Seeded dissolution tests

For most glass compositions, the initiation time before Stage III behavior is quite long, often measured in years, even in 

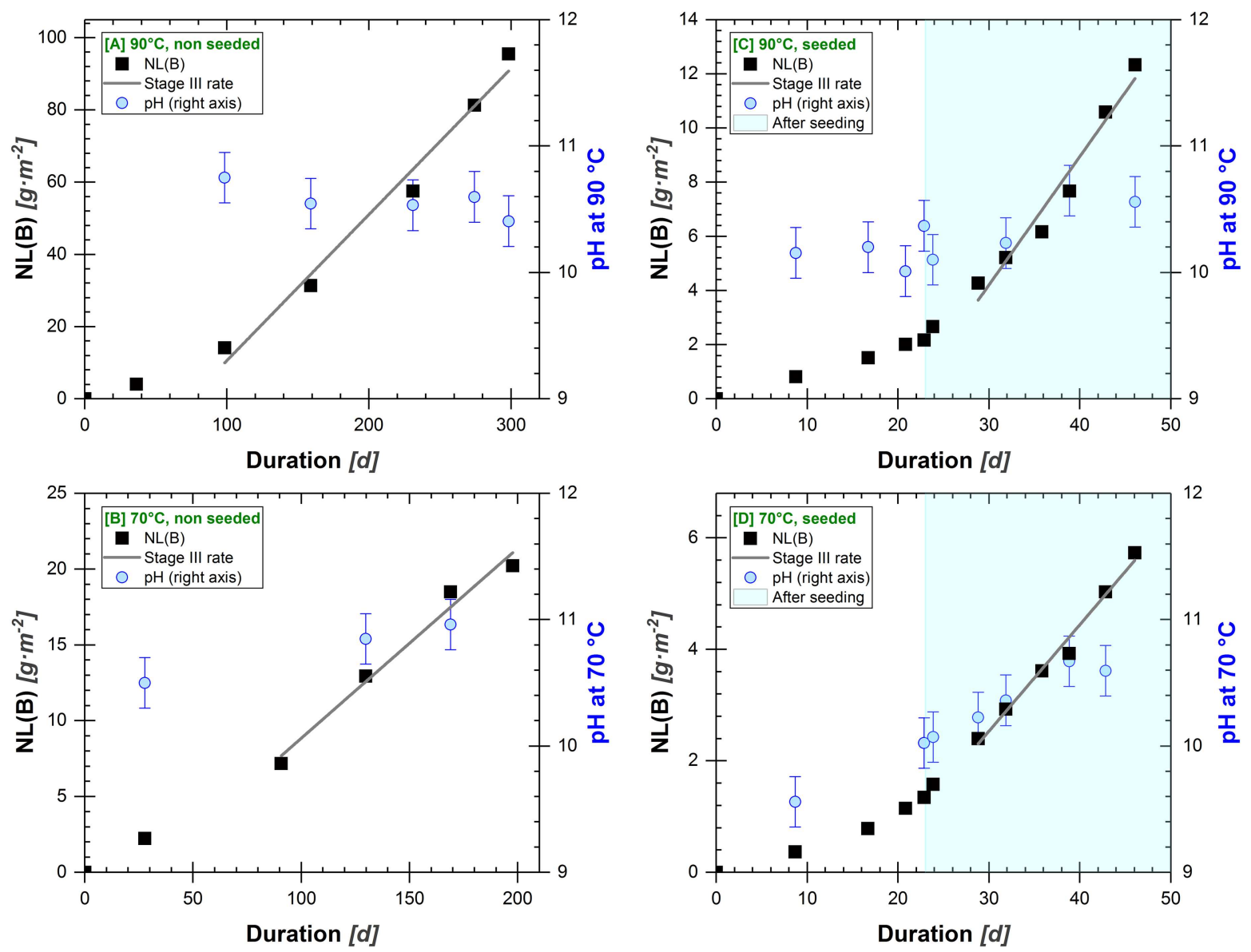

Fig. 2 Solution analysis data of boron (left axis) and pH (right axis) for static dissolution tests on LAWA76 glass. Left: Tests in initially ultrapure water with NO zeolite addition: $\mathbf{a}$ at $90^{\circ} \mathrm{C}, \mathbf{b}$ at $70^{\circ} \mathrm{C}$. Right: Tests in initially ultrapure water where Na-P2 zeolite seeds were added to each experiment after 23 days (denoted by the blue-shaded regions on the right side of each plot): $\mathbf{c}$ at $90^{\circ} \mathrm{C}, \mathbf{d}$ at $70^{\circ} \mathrm{C}$

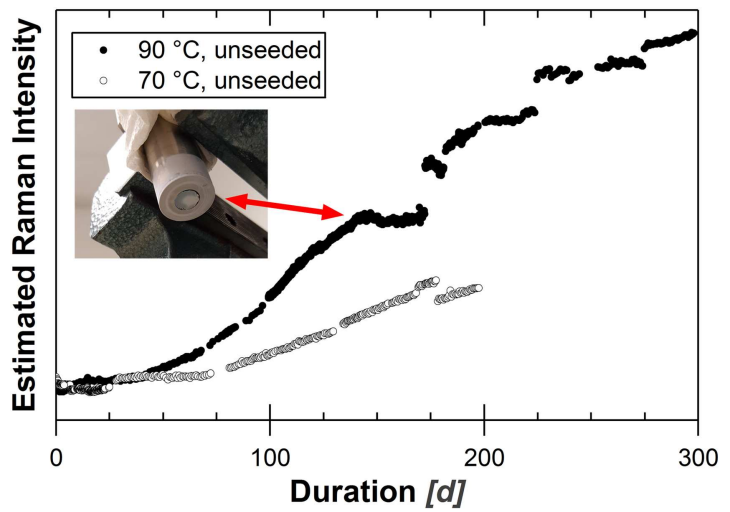

Fig. 3 Raman monitoring of LAWA76 glass at $90^{\circ} \mathrm{C}$ and $70^{\circ} \mathrm{C}-$ unseeded. Deviations of the Raman signal during $90^{\circ} \mathrm{C}$ test were because of zeolite precipitation on the probe window (inset)

accelerated conditions. As mentioned earlier, this makes test design difficult and sampling intervals non-optimal. Seeds of premade zeolites that are the same composition as those observed during Stage III behavior can enable the measurement of Stage III rates with accuracy at reasonable times. ${ }^{8}$ This approach is predicated, however, on the assumption that the dissolution rates in un-triggered conditions are equivalent to those observed following seeding.
To test this assumption, seeds were added to static dissolution experiments at $90^{\circ} \mathrm{C}$ and $70^{\circ} \mathrm{C}$ identical to those run for the unseeded tests (Fig. 2) after 23 days. In the initial unseeded testing results, it appeared that Stage II dissolution rates had largely been achieved by this point but Stage III had not initiated. Solution analysis during the portion of the experiments before seeding confirmed a much lower rate in each experiment. The introduction of the Na-P2 zeolite seeds resulted in a prompt increase in dissolution behavior very similar to the onset of Stage III dissolution observed in unseeded experiments after $\sim 80$ days. Solution analyses gave a range of Stage III dissolution rates from 0.47 to $0.62 \mathrm{~g} \mathrm{~m}^{-2} \mathrm{~d}^{-1}$ for the $90^{\circ} \mathrm{C}$ seeded experiments, and a value of $0.19 \mathrm{~g} \mathrm{~m}^{-2} \mathrm{~d}^{-1}$ for the $70^{\circ} \mathrm{C}$ seeded experiments. Because seeding allowed control of the onset of Stage III behavior, it was possible to conduct more frequent chemical analysis when it was most needed.

Chemometric analyses

The results of the validation for the chemometric modelling of the $\mathrm{B}$ concentration in solution and of the $\mathrm{pH}$ are presented in Fig. 4. Figure $4 a$ presents a parity plot comparing chemometrically measured results to known values for both $\mathrm{B}$ (top) and $\mathrm{pH}$ (bottom). In each case, results from the training set (used to build the model) and the validation sets (spectra from the seeded and unseeded experiments) are presented. Locally weighted regression models were utilized to build robust models capable of tolerating the non-linear temperature and $\mathrm{pH}$ effects. ${ }^{14}$ Preprocessing included applying a first derivative, normalization to water 
A
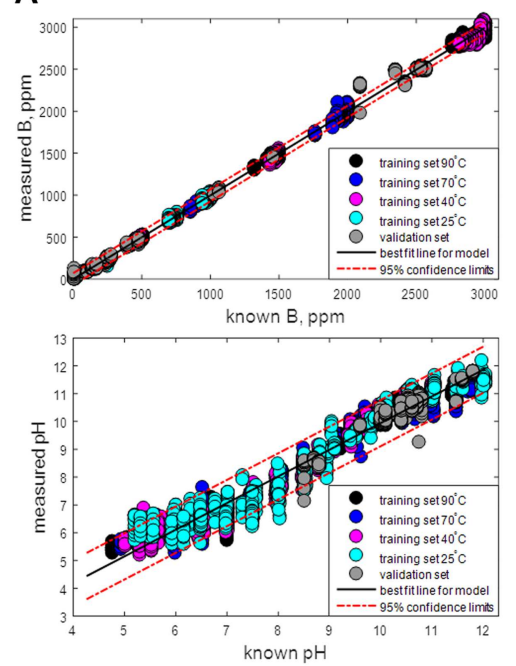

B
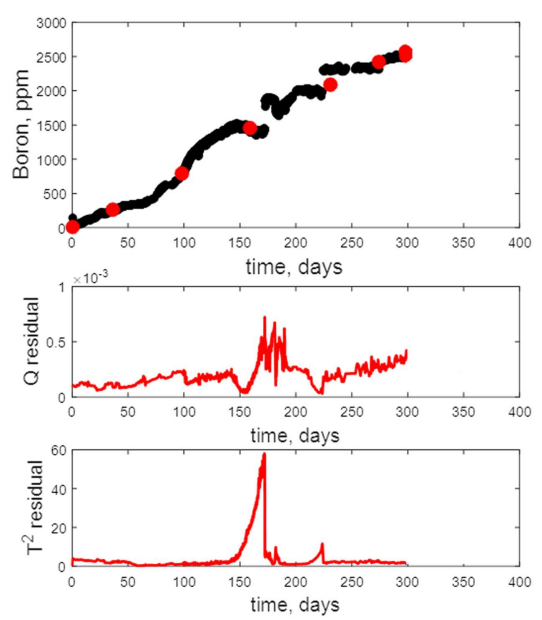

C
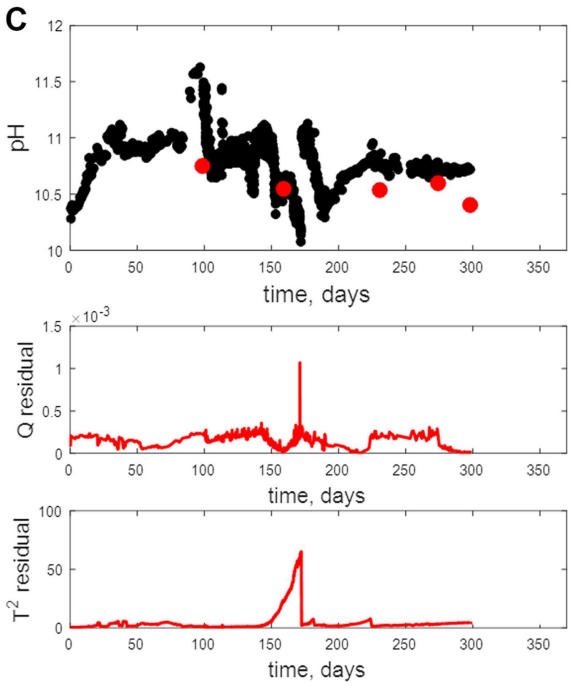

Fig. 4 a Chemometric training set correlation plots for B concentration (top) and $\mathrm{pH}$ (bottom). b Boron concentrations in solution (top) derived from in situ chemometric Raman analysis (black) and solution analyses (red) for the $90^{\circ} \mathrm{C}$ unseeded static dissolution test, together with $\mathrm{Q}$ residual (middle) and $\mathrm{T}^{2}$ residual (bottom). c Chemometric analysis (black) and direct measurement results (red) of the pH during the $90^{\circ} \mathrm{C}$ unseeded static dissolution test (top), together with $\mathrm{Q}$ residual (middle) and $\mathrm{T}^{2}$ residual (bottom). $\mathrm{Q}$ residual and $\mathrm{T}^{2}$ residual are inversely proportional to the goodness of fit for any particular spectrum, and both indicate the correlation between model value variability and corresponding high residuals

band, and mean center to account for variations in baselines, signal intensity, and model weightings respectively. The fits for these calibrations were good as indicated by tight the $95 \%$ confidence limits, with some expected outliers predominantly from the seeded and unseeded experiments, likely caused by spectral interferences from zeolite formation. These data points exhibit high residuals (indicating the sample does not conform to the model) and should therefore not be considered representative. ${ }^{15}$ Both $\mathrm{Q}$ and Student $\mathrm{T}^{2}$ residuals were utilized to examine data quality. The $\mathrm{pH}$ model exhibits less ideal behavior, appearing to take on a more non-linear character, particularly at low temperatures. Based on previous studies, this model could be improved by expanding the training set to better capture spectral variance across the temperature ranges. ${ }^{16,17}$

The boron concentrations obtained through the chemometric modeling of in situ Raman data matched the solution analysis data extremely well (Fig. 4b). At every point, the Raman-derived and solution derived values differed by less than $5 \%$. This is well within the expected error for the solution analyses themselves. The high temporal coverage of the Raman measurements fills in the time gaps between the discrete ICP-OES solution analysis measurements. The existence of slower Stage II behavior during the 35-60 day time period can be clearly observed. The data collection issues due to the coverage of the probe window were also evident. In this case, however, spectral data heavily compromised by window coverage can be quickly identified based on high residual values.

As expected from the training set results, the model-derived $\mathrm{pH}$ values showed limited precision and less ideal agreement with the values measured via $\mathrm{pH}$ probe. Through the duration of highest glass alteration, high $\mathrm{Q}$ and $\mathrm{T}^{2}$ residuals were observed, indicating disparity between spectral features observed in the training sets and actual experimental data. Similar trends in residual behavior were observed for both boron and $\mathrm{pH}$ measurements. Again this can be correlated to zeolite formation and the blocking of the spectral window (Fig. 4c). It is possible to set cutoff limits on residuals to remove poor quality data, but in this instance cut offs were not applied to allow for the demonstration of measurement/ residual trends. It appeared that the experimentally-measured values matched the modeled values for each experimental measurement point within $0.3 \mathrm{pH}$ units; however, the variability of the results discourages their use for analyzing the experiment until more robust models are generated.

\section{DISCUSSION}

To this point, and even including this study, Stage III behavior has only been observed for tests performed in highly aggressive conditions at high $\left(\geq 90^{\circ} \mathrm{C}\right)$ temperatures. These conditions bear little resemblance to the environments of potential nuclear waste glass repositories, where low temperature, low $S A N$, and fluid renewal can all serve to depress the likelihood, initiation, and rate of Stage III dissolution. Despite those caveats, the dramatic acceleration of Stage III behavior is potentially impactful such that it is important to increase the understanding of Stage III dissolution behavior in relevant environmental conditions.

The delay before the commencement of Stage III dissolution behavior complicates the examination of this mechanism. It is thought that the delay is due to the development of critical nuclei that then can rapidly grow in a supersaturated solution. ${ }^{8}$ If this is the case, it is unlikely that the delay could ever be accurately predicted. In fact, it is unknown whether the delay period would remain the same in identical tests. Two equivalent tests on the LAWA44 glass seemed to show a dramatic difference in the delay period before Stage III and one test did not appear to trigger at all despite more than a 3.5 year period of record. ${ }^{9}$ Thus, the random nature of most nucleation processes ${ }^{18}$ would make the prediction of any incubation period an exercise in probability, at best. Instead, it is more appropriate to focus on whether the critical phases could form, and what the impacts would be, if they did.

In a sense, the questions of whether the phases would form and what their impacts would be are reflected in the competition between thermodynamic and kinetic controls. A common artificial synthesis pathway for key zeolite phases is to utilize glass and some of the more innocuous alteration products such as calciumsilicate-hydrate, ${ }^{19,20}$ suggesting that those transitions are thermodynamically favored. This is why an analysis of the temperaturedependence of this behavior is critical. The results here 


\begin{tabular}{|lc|}
\hline Table 1. & Composition of LAWA76 in mol\% \\
\hline Oxide & mol\% \\
\hline $\mathrm{SiO}_{2}$ & 44.26 \\
$\mathrm{~B}_{2} \mathrm{O}_{3}$ & 9.95 \\
$\mathrm{Na}_{2} \mathrm{O}$ & 10.34 \\
$\mathrm{Al}_{2} \mathrm{O}_{3}$ & 3.82 \\
$\mathrm{CaO}$ & 8.85 \\
$\mathrm{Cl}$ & 1.16 \\
$\mathrm{Cr}_{2} \mathrm{O}_{3}$ & 0.008 \\
$\mathrm{~F}$ & 0.033 \\
$\mathrm{Fe}_{2} \mathrm{O}_{3}$ & 2.96 \\
$\mathrm{~K}_{2} \mathrm{O}$ & 0.335 \\
$\mathrm{Li}_{2} \mathrm{O}$ & 10.58 \\
$\mathrm{MgO}$ & 2.31 \\
$\mathrm{SO}_{3}$ & 0.682 \\
$\mathrm{TiO}_{2}$ & 0.874 \\
$\mathrm{ZnO}^{\mathrm{ZrO}_{2}}$ & 2.32 \\
\hline
\end{tabular}

conclusively show that Stage III behavior is possible at temperatures as low as $70^{\circ} \mathrm{C}$. Given the rapid initiation of the behavior, this temperature is not likely to be close to the lower limit, although this was not tested in this effort. This is not surprising, given that in natural analogue systems, zeolites have been observed to form at temperatures as low as $5{ }^{\circ} \mathrm{C}$ on the surfaces of basaltic glasses $10^{5}$ years old and older. ${ }^{21,22}$ The conditions under which these ancient basaltic glasses were subjected to are markedly different from the glasses and conditions studied here, but this provides tangential evidence that the thermodynamics likely do not preclude the formation of at least some zeolites at low temperatures.

The most immediate and tractable portion of the Stage III mechanism to study is the kinetics of the process after initiation. Seeding has been shown to accelerate the initiation of Stage III behavior. Assuming the mechanism is the same in seeded and unseeded tests, this enables a more detailed analysis of the mechanism. The Stage III rates observed for the $90^{\circ} \mathrm{C}$ experiments were essentially equivalent for both unseeded and seeded experiments (e.g., $0.40-0.56 \mathrm{~g} \mathrm{~m}^{-2} \mathrm{~d}^{-1}$ and $0.47-0.62 \mathrm{~g}$ $\mathrm{m}^{-2} \mathrm{~d}^{-1}$, respectively). This equivalency was also seen in the $70{ }^{\circ} \mathrm{C}$ rates, with $0.13 \mathrm{~g} \mathrm{~m}^{-2} \mathrm{~d}^{-1}$ for the unseeded experiments and $0.19 \mathrm{~g} \mathrm{~m}^{-2} \mathrm{~d}^{-1}$ for the seeded experiments. While this should be confirmed for additional glasses and temperatures, it appears that seeding is able to expedite the onset of Stage III dissolution and is able to induce similar dissolution behavior without introducing experimental artifacts. The decrease in rate with temperature also suggests that the system activation energy may be determinable with additional testing. If the activation energy can be obtained, the rate, and thus impact, of Stage III dissolution could be determined for multiple potential repository environments.

The ability to track the general evolution of the boron signal using in situ Raman monitoring brought a new level of understanding to the experiment. Without this technique the brief Stage II dissolution regime would have been missed. Further, the technique improved control and fidelity of the experiment sampling. The number of ICP-OES samples was reduced compared to what is generally done for similar experiments and were targeted to sample the most important time periods. This had the benefit of reducing perturbations to the experiment caused by
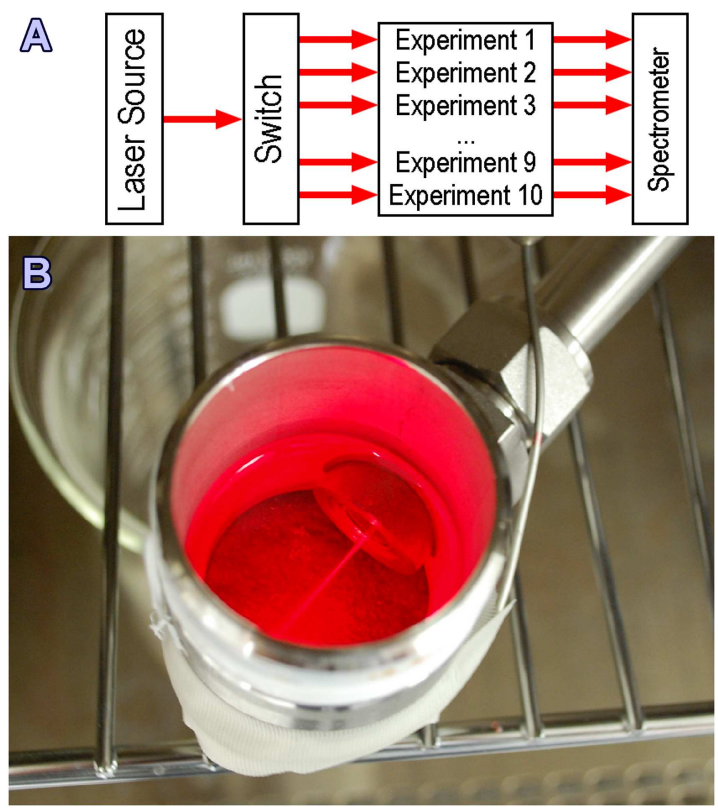

Fig. 5 Schematic (a) of the in situ Raman monitoring system and image (b) of the sample reactor

sampling, including less contamination from $\mathrm{pH}$ measurements with a glass electrode, reduced temperature variations endured during sampling, and less solution volume variations due to sampling, and less evaporation during sampling. Sudden signal variations prompted investigation into the general status of the alteration vessel and led to the observation of solid precipitation on the entire surface of the alteration vessel, including the window of the Raman probe. This information supports the mechanistic theory that Stage III occurs through solution feedback.

While in situ, real-time Raman signal measurement is a valuable way to monitor the progress of solution chemistry changes, the simple method used here does not provide quantitative $\mathrm{pH}$ values and concentrations of boron in solution like chemometric modelling does. The data obtained on the $90^{\circ} \mathrm{C}$ unseeded experiment is promising and follows the general trends observed through ICP-OES sampling and $\mathrm{pH}$ measurement in the vessel. However, more development is necessary to reduce the overall error and better understand/mitigate the effect of interferences such as zeolite formation. This will be the topic of future papers.

This research demonstrates that Stage III glass corrosion behavior is possible at temperatures below $90^{\circ} \mathrm{C}$. While results on tests at temperatures lower than $70^{\circ} \mathrm{C}$ were not available for this study, a comprehensive examination of the differences in Stage III dissolution rates with temperature is warranted. The confirmation in this study that seeded and unseeded Stage III corrosion rates are equivalent suggests a similarity, if not equivalence, in mechanism and opens the possibility to perform wide-scale testing of Stage III corrosion rates for many glass compositions in relatively short times. Further, in situ monitoring of boron concentration and $\mathrm{pH}$ is a powerful tool for examining variations in Stage III behavior. Recent works highlight how solution $\mathrm{pH}$ and related components are major determining factors for whether Stage III occurs. ${ }^{9,23,24}$ More details will enable the inclusion of this impactful mechanism into long-term corrosion models and improve the estimates of long-term waste form performance in a variety of repository conditions. 


\section{METHODS}

Glass preparation

A glass designed for low-activity waste immobilization, termed LAWA76, was chosen for its propensity to exhibit Stage III behavior with a short incubation time (Fig. 1b). The glass composition (in mol\%) is given in Table 1. This glass was produced by mixing oxides, carbonates, and $\mathrm{H}_{3} \mathrm{BO}_{3}$ in an agate mill, melting in a covered $\mathrm{Pt} / 10 \% \mathrm{Rh}$ crucible at $1150^{\circ} \mathrm{C}$ for $1 \mathrm{~h}$, quenching on an Inconel ${ }^{\circ}$ pour plate, grinding in a tungsten carbide mill, remelting under the same conditions as before, and requenching. Quenched glass was ground, sieved (75-150 $\mu \mathrm{m}$ (100-200 mesh) powder size fraction), and washed several times to eliminate fines following ASTM procedure $\mathrm{C} 1285-14 .^{25}$

\section{Zeolite Na-P2 (seed) preparation}

Experiments at $70^{\circ} \mathrm{C}$ and $90^{\circ} \mathrm{C}$ performed with equivalent conditions to those above were seeded with zeolites after solution analysis confirmed that the tests were in Stage II (residual rate) conditions. The zeolite Na-P2 used for this experiment was synthesized by a procedure reported previously. ${ }^{26}$ Sodium hydroxide or potassium hydroxide was dissolved in deionized water to which aluminum sulfate hydrate or sodium aluminate was added. The synthesis mixture was stirred until it became clear. To this mixture, LUDOX AS-40 colloidal silica was then slowly added as the silica source under continuous stirring. The resulting solution was stirred at room temperature for approximately $22-24 \mathrm{~h}$. The solutions were transferred to a Teflon liner, which was capped and placed in a stainless steel autoclave and heated to $100^{\circ} \mathrm{C}$ for 7 days. After cooling down to room temperature, the solid crystals were recovered by centrifugation followed by washing three times with deionized water and the product was dried in air at room temperature. X-ray diffraction analysis (not shown) confirmed the structure and purity. The median particle size was $12 \mu \mathrm{m}$, determined using a Malvern Mastersizer 2000 analyzer and used for surface-area calculations.

\section{Dissolution tests}

Alteration experiments were performed in static conditions at several temperatures in 304 stainless steel reactors specifically designed to accommodate the Raman probe for in situ measurement (Fig. 5b). Samples were corroded in the measurement vessels with $>18.0 \mathrm{M} \Omega \cdot \mathrm{cm}$ water (ASTM type $^{27}$ ) at $2000 \mathrm{~m}^{-1} \mathrm{SA} / \mathrm{V}$. The $\mathrm{pH}$ values of the experiment solutions were periodically measured at test temperature directly in the alteration vessel and sample aliquots were taken to monitor elemental concentrations in solution and track corrosion process. All aliquots were diluted using $0.3 \mathrm{~mol} / \mathrm{L}$ nitric acid prepared from trace metal grade $70 \% \mathrm{HNO}_{3}$. The mass balance of the water in the vessel was controlled periodically, with evaporated water replenished using ASTM type I water to match the target mass of water at that time (initial mass of water minus all previous aliquot masses).

Two experiments performed with equivalent conditions to those above were seeded with zeolite $\mathrm{Na}-\mathrm{P} 2$ after solution analysis confirmed that the tests were in Stage II (residual rate) conditions. The amount added was adjusted so that the external geometric $S A / V$ ratio of zeolite added to each seeded test was $1000 \mathrm{~cm}^{-1}$. Considering the volume of the reaction vessels, $208 \mathrm{mg}$ of zeolite Na-P2 were added 23 days after each test was initiated.

\section{Data treatment and dissolution tracking}

The normalized mass loss of boron at time $t$, or $N L_{B}(t)$, was used to track glass dissolution and was determined using Equation 1 below:

$N L_{B}(t)=\frac{m_{B, t}}{f_{B} \times S_{\text {glass }, t}}=\frac{[B]_{t} \times V_{\text {vessel }, t}+\sum_{i=1}^{t}\left([B]_{t_{i}} \times V_{\text {sampled }, t_{i}}\right)}{f_{B} \times S_{\text {glass }, t}}$

where $N L_{B}(t)$ is the normalized mass loss of the glass based on the $B$ concentration in solution at time $t\left(g_{\text {glass }} \cdot \mathrm{m}^{-2}\right) ; m_{B, t}$ is the total mass of $B$ released by the glass at time $t(\mathrm{~g}) ; f_{B}$ is the mass fraction of $\mathrm{B}$ in the glass (unitless); $[B]_{t}$ is the concentration of $B$ at time $t\left(\mathrm{~g} \cdot \mathrm{m}^{-3}\right) ; V_{\text {vessel, }}$ is the volume of solution in the alteration vessel at time $t$ after sampling $\left(\mathrm{m}^{3}\right)$; $V_{\text {sampled, } t}$ is the volume of solution sampled at time $t\left(\mathrm{~m}^{3}\right)$; and $S_{\text {glass, } t}$ is the surface area of the glass-solution interface at time $t$ calculated using the shrinking core model $\left(\mathrm{m}^{2}\right.$, see Equation 2 below):

$S_{\text {glass }, t}=\frac{3}{R_{0} \times \rho} \times m_{\text {glass }, t}^{2 / 3} \times m_{\text {glass }, 0}^{1 / 3}=\frac{3}{R_{0} \times \rho} \times\left(m_{\text {glass }, 0}-\frac{m_{B, t}}{f_{B}}\right)^{2 / 3} \times m_{\text {glass }, 0}^{1 / 3}$

where $R_{0}$ is the initial diameter of the glass particles $(\mathrm{m}) ; \rho$ is the density of the glass $\left(\mathrm{g} \cdot \mathrm{m}^{-3}\right) ; m_{\text {glass }, t}$ is the remaining mass of unaltered glass at time $\mathrm{t}$ (g); and $m_{\text {glass,o }}$ is the initial mass of unaltered glass (g).

\section{In situ Raman monitoring}

The Raman monitoring system used in this study was purchased from Spectra Solutions Inc. and is capable of sequentially monitoring 10 different experiments (Fig. 5a). It is composed of: a $450 \mathrm{~mW}$ visible $671 \mathrm{~nm}$ diode laser used as an excitation source and a 10-channel optical switch directing the laser beam sequentially to each of the Raman probes connected to its output ports. Acquisition times were optimized for signal to noise ratio and typically fell in the realm of $10 \mathrm{~s}$. Preprocessing of the Raman data, prior to model building and application, included normalizing to account for laser power/acquisition time fluctuations as well as baseline correction. This is discussed in more detail in the modeling section. The Raman probes were built by Spectra Solutions and were affixed to specifically designed vessels (Fig. 5b) to allow direct measurement of the solution. The probes are enclosed in a stainless steel sleeve equipped with a quartz window from which the laser beam focal point is $1 \mathrm{~cm}$ inside the solution. Raman measurements were performed with a SPR-M671 spectrograph containing a thermoelectrically-cooled charged coupled device (CCD) detector operating at $-55^{\circ} \mathrm{C}$. All elements are optically interconnected using $50 \mu \mathrm{m}$ diameter optical fibers. The Spectrasoft Multi software (Spectra Solutions Inc.) was used to control the instrument and acquire spectra. The acquisition duration was adjusted for each channel and for each new sequence of data acquired to maximize the signal received by the detector.

A qualitative treatment of the Raman spectra was performed as the experiment was ongoing. For this analysis 100 acquired spectra were averaged and normalized to the area of the $\mathrm{OH}$ stretching band $\left(3000-3600 \mathrm{~cm}^{-1}\right)$ before the height of the $745 \mathrm{~cm}^{-1} \mathrm{~B}(\mathrm{OH})_{4}^{-}$symmetric stretch peak ${ }^{28}$ was extracted. The $\mathrm{B}(\mathrm{OH})_{3}$ symmetric stretch peak at $870 \mathrm{~cm}^{-1}$ was ignored as when $\mathrm{pH}_{90^{\circ} \mathrm{C}}>10.05, \mathrm{~B}(\mathrm{OH})_{4}{ }^{-}$predominated at more than $95 \%$. Although limited and only qualitative, this day-to-day data analysis allowed for educated and limited ICP-OES samplings based on a first glance at processes occurring in the alteration vessel.

The same general procedure detailed by Parruzot et al. ${ }^{16}$ was used to acquire the Raman chemometric data for all tests detailed in this article. Chemometric modeling is a form of multivariate analysis that allows for the quantification of target species or properties. ${ }^{17,29-31}$ Here we use chemometric analysis to quantify total $\mathrm{B}$ concentration as well as $\mathrm{pH}$. Models are built from spectroscopic training sets, which were processed using the PLS_Toolbox (Eigenvector Research Inc.) in MATLAB (MathWorks R2015b). ${ }^{15}$ The training set used to build the chemometric model included three solutions of variable $B$ concentration ( 250 to $3000 \mathrm{ppm} \mathrm{B}$ ) that were analyzed at four relevant test temperatures $\left(90^{\circ} \mathrm{C}, 70^{\circ} \mathrm{C}, 40^{\circ} \mathrm{C}\right.$, and room temperature). The $\mathrm{pH}$ of each solution $\left(\mathrm{pH}_{\text {Test }} \mathrm{T}^{\circ} \mathrm{C}\right)$ was varied using $\mathrm{NaOH}$ (99.99\% purity grade) between 4.7 and 12.0. The training sets were collected at roughly the same range of acquisition times as those used in the experiment monitoring. Model details and performance parameters are described in the results and discussion sections.

\section{DATA AVAILABILITY}

The datasets generated and analyzed during the current study are available from the corresponding author on reasonable request.

\section{ACKNOWLEDGEMENTS}

This work was jointly funded by the US Department of Energy Office of Nuclear Energy (Materials Recovery and Waste Form Development) and the Office of Environmental Management (Tank Waste Management, EM-21). Pacific Northwest National Laboratory is a multi-program national laboratory operated for the U.S. Department of Energy by Battelle Memorial Institute under Contract DEAC06-76RLO 1830. 


\section{AUTHOR CONTRIBUTIONS}

J.V.R. and B.P. coordinated the study, performed the data analysis, and wrote the manuscript. A.L. and S.A.B. performed the in situ Raman data analysis and contributed to the writing of the manuscript. L.M.S. and J.F.B. performed the experimental work for the study and contributed to the data analysis. R.K.M. synthesized and characterized the zeolites.

\section{ADDITIONAL INFORMATION}

Competing interests: The authors declare no competing interests.

Publisher's note Springer Nature remains neutral with regard to jurisdictional claims in published maps and institutional affiliations.

\section{REFERENCES}

1. Van Iseghem, P. \& Grambow, B. The Long-Term Corrosion and Modelling of Two Simulated Belgian Reference High-Level Waste Glasses. (Scientific Basis for Nuclear Waste Management XI, Pittsburgh, Pennsylvania, 1988).

2. Papathanassiu, A. et al. ILAW Glass Testing for Disposal at IDF: Phase 1 Testing, Vitreous State Laboratory. (The Catholic University of America, VSL-11R2270-1, 2011).

3. Pierce, E. M. et al. Accelerated weathering of high-level and plutonium-bearing lanthanide borosilicate waste glasses under hydraulically unsaturated conditions. Appl. Geo. 22, 1841-1859 (2007).

4. Bacon, D. H. \& Pierce, E. M. Sensitivity Analysis of Kinetic Rate-Law Parameters Used to Simulate Long-Term Weathering of ILAW Glass. (PNNL-19472, Pacific Northwest National Laboratory, Richland, Washington, 2010).

5. Ribet, S. et al. Compositional effects on the long-term durability of nuclear waste glasses: a statistical approach. MRS Online Proc. Libr. Arch. 824, CC5.3 (2004)

6. Ribet, S. \& Gin, S. Role of neoformed phases on the mechanisms controlling the resumption of SON68 glass alteration in alkaline media. J. Nucl. Mater. 324, 152-164 (2004).

7. Strachan, D. M. \& Croak, T. L. Compositional effects on long-term dissolution of borosilicate glass. J. Non-Cryst. Solids 272, 22-33 (2000).

8. Fournier, M. et al. Effect of zeolite formation on borosilicate glass dissolution kinetics. Procedia Earth Planet. Sci. 7, 264-267 (2013).

9. Jantzen, C. M. et al. Accelerated Leach Testing of GLASS (ALTGLASS): I. Informatics approach to high level waste glass gel formation and aging. Int. J. Appl. Glass Sci. 8, 69-83 (2017).

10. Ebert, W. L. et al. Measurement of the Glass Dissolution Rate in the Presence of Alteration Phases, from Proceedings International Topical Meeting on Nuclear and Hazardous Waste Management. (Spectrum ‘96, Seattle, 1996).

11. Wronkiewicz, D. J. \& Arbesman, K. A. The role of alteration phases in influencing the kinetics of glass dissolution. MRS Proc. 608, 745 (1999).

12. Fournier, M. Etude des mécanismes à l'origine des reprises d'altération: Modélisation et impact sur les verres de confinement [Study of the mechanisms underlying resumptions of alteration: Modeling and evaluation of the impact on nuclear waste glasses], Thesis, Université Montpellier, (2015).

13. Taylor, A. M. \& Roy, R. Zeolite studies iv: Na-P zeolites and the ion-exchanged derivatives of tetragonal Na-P1. Am. Mineral. 49, 656-682 (1964).

14. Ruppert, D. \& Wand, M. P. Multivariate locally weighted least-squares regression. Ann. Stat. 22, 1346-1370 (1994).

15. Wise, B. M. et al. Manual PLS_Toolbox, Version 4.0. (Eigenvector Research Inc, Wenatchee, 2006).
16. Parruzot, B. et al. Method for the in situ measurement of $\mathrm{pH}$ and alteration extent for aluminoborosilicate glasses using Raman spectroscopy. Anal. Chem. 90, 11812-11819 (2018).

17. Casella, A. J. et al. Development of online spectroscopic pH monitoring for nuclear fuel reprocessing plants: weak acid schemes. Anal. Chem. 87, 5139-5147 (2015).

18. Siclen, C. D. Van Random nucleation and growth kinetics. Phys. Rev. B 54, 11845-11848 (1996).

19. H. Ghobarkar, H. et al. The Reconstruction of Natural Zeolites. (Kluwer Academic Publishers, Boston, 2003).

20. Mostafa, N. Y., Garib, R. A., Heiba, Z. K., Abd-Elkader, O. H. \& Al-Majthoub, M. M. Synthesis of pure zeolite $\mathrm{P} 2$ from calcium silicate hydrate tobermorite. Orient $\mathrm{J}$. Chem. 31, (2015). https://doi.org/10.13005/ojc/310254.

21. Crovisier, J. L. et al. Nature and role of natural alteration gels formed on the surface of ancient volcanic glasses (Natural analogs of waste containment glasses). J. Nucl. Mater. 321, 91-109 (2003).

22. Parruzot, B. Altération des verres basaltiques dans des environnements confinés: analogie avec le stockage géologique des verres nucléaires [Basaltic glass alteration in confined environments: analogy to nuclear waste glass geological repository]. Thesis, Université Montpellier 2, (2014).

23. Jantzen, C. M. et al. Accelerated Leach Testing of Glass (ALTGLASS): II. Mineralization of hydrogels by leachate strong bases. Int. J. Appl. Glass Sci. 8, 84-96 (2017).

24. Trivelpiece, C. L. et al. Corrosion of ISG fibers in alkaline solutions. J. Am. Ceram. Soc. 100, 4533-4547 (2017).

25. ASTM C1285-14. Standard Test Methods for Determining Chemical Durability of Nuclear, Hazardous, and Mixed Waste Glasses and Multiphase Glass Ceramics: The Product Consistency Test (PCT). (ASTM International, West Conshohocken, 2014).

26. Oleksiak, M. D. et al. Synthesis strategies for ultrastable zeolite GIS polymorphs as sorbents for selective separations. Chem. A Eur. J. 22, 16078-16088 (2016).

27. ASTM D1193-06. Standard Specification for Reagent Water. (ASTM International, West Conshohocken, 2018).

28. George, J. L. \& Brow, R. K. In-situ characterization of borate glass dissolution kinetics by $\mu$-Raman spectroscopy. J. Non-Cryst. Solids 426, 116-124 (2015).

29. Beebe, K. R. et al. Chemometrics: A Practical Guide. (Wiley, New York, 1998).

30. Bro, R. \& Elden, L. PLS works. J. Chemom. 23, 69-71 (2009).

31. Lines, A. M. et al. Multivariate analysis for quantification of plutonium (IV) in nitric acid based on absorption spectra. Anal. Chem. 89, 9354-9359 (2017).

(c) Open Access This article is licensed under a Creative Commons cc Attribution 4.0 International License, which permits use, sharing, adaptation, distribution and reproduction in any medium or format, as long as you give appropriate credit to the original author(s) and the source, provide a link to the Creative Commons license, and indicate if changes were made. The images or other third party material in this article are included in the article's Creative Commons license, unless indicated otherwise in a credit line to the material. If material is not included in the article's Creative Commons license and your intended use is not permitted by statutory regulation or exceeds the permitted use, you will need to obtain permission directly from the copyright holder. To view a copy of this license, visit http://creativecommons. org/licenses/by/4.0/.

This is a U.S. government work and not under copyright protection in the U.S.; foreign copyright protection may apply 2019 